\title{
Kuhn, Christian, Generation als Grundbegriff einer historischen Geschichtskultur. Die Nürnberger Tucher im langen 16. Jahrhundert
}

\section{Aude-Marie Certin}

\section{OpenEdition}

Édition électronique

URL : http://journals.openedition.org/ifha/6654

DOI : 10.4000/ifha.6654

ISSN : 2198-8943

Éditeur

IFRA - Institut franco-allemand (sciences historiques et sociales)

\section{Référence électronique}

Aude-Marie Certin, « Kuhn, Christian, Generation als Grundbegriff einer historischen Geschichtskultur. Die Nürnberger Tucher im langen 16. Jahrhundert », Revue de l'IFHA [En ligne], Date de recension, mis en ligne le 01 janvier 2011, consulté le 22 septembre 2020. URL : http://journals.openedition.org/ifha/ 6654 ; DOI : https://doi.org/10.4000/ifha.6654

Ce document a été généré automatiquement le 22 septembre 2020.

(CIFHA 


\title{
Kuhn, Christian, Generation als Grundbegriff einer historischen Geschichtskultur. Die Nürnberger Tucher im langen 16. Jahrhundert
}

\author{
Aude-Marie Certin
}

Issu d'une thèse de doctorat soutenue à Bamberg en 2007, cet ouvrage de C.K. se propose de montrer toute la valeur heuristique du concept de génération pour comprendre la culture historique des élites patriciennes du XVIe siècle, à travers le cas exemplaire des Tucher de Nuremberg.

Dans la première partie introductive, l'auteur définit le cadre conceptuel et historique de son analyse. La réflexion de C.K. sur la génération articule de fait deux perspectives ; elle approfondit d'une part la conception sociologique de Karl Mannheim qui, dans une optique synchronique, définit la génération comme une communauté d'expérience ; d'autre part, elle adopte une approche plus diachronique et dynamique du concept, prenant davantage en compte la question de la transmission entre les groupes d'âge.

Pour mener à bien cette réflexion, C.K. se base sur les différentes sources textuelles et matérielles laissées tout au long du XVIe siècle par la famille patricienne des Tucher. Dans un premier temps, l'auteur étudie les relations entre les générations dans les nombreuses lettres échangées entre certains pères de la famille, notamment Anton et Leonhard Tucher, et leur fils, au moment des années de formation de ces derniers à l'étranger. Et l'auteur de montrer qu'au-delà de leur portée éducative explicite, ces lettres prennent aussi sens dans une perspective mémorielle. Écrites et conservées avec soin, elles visent de fait à perpétuer de père en fils les normes et les valeurs au fondement de l'identité familiale.

Dans un second temps, C.K. étudie les différentes versions du Grosse Tucherbuch, du premier livre de famille élaboré par Christoph Scheurl en 1542 jusqu'aux somptueuses versions datant de la fin du XVIe siècle. En analysant la dimension matérielle, textuelle et iconographique de ces textes, C.K révèle les évolutions dans la façon de penser 
l'histoire de la famille tout au long du XVIe siècle. Alors que la première version du Tucherbuch met en valeur l'histoire prestigieuse de la famille, les versions ultérieures du livre de lignage visent davantage à affirmer l'unité de la famille dans le temps. C.K. montre ainsi comment les Tucher cherchent à asseoir leurs privilèges patriciens et la continuité de leur pouvoir dans un contexte d'importants changements sociaux, politiques et confessionnels.

Depuis le milieu des années 2000, la Generationenforschung constitue un champ de réflexion particulièrement dynamique de l'histoire allemande. En témoignent les différents groupes de recherches travaillant sur cette question, de l'époque antique à l'époque contemporaine. L'ouvrage de C.K. s'inscrit dans ce mouvement historiographique et son grand apport est de proposer un modèle d'interprétation général du concept de génération. Par là même, il ouvre des perspectives d'analyse extrêmement stimulantes, notamment pour l'étude de l'histoire du patriciat au XVIe siècle.

Aude-Marie Certin (IFHA) 